\title{
Impact of Live Training on Medication Errors in a Community-Based Pharmacy Setting
}

Morgan Grant, PharmD ${ }^{*}$; Jamie Remines, PharmD²; Pramit Nadpara, PhD, MS, BPharm³; Jean-Venable "Kelly" R. Goode, PharmD, BCPS, FAPhA, FCCP ${ }^{3}$

${ }^{1}$ Kroger Pharmacy; ${ }^{2}$ Academia SME, Kroger Health \& Wellness - Mid-Atlantic; ${ }^{3}$ Virginia Commonwealth University School of Pharmacy

*At time of writing, PGY1 Community-Based Pharmacy Practice Resident of the Virginia Commonwealth University School of Pharmacy and Kroger Pharmacy.

\section{Abstract}

Objective: To compare community-based pharmacy medication errors before and after a one-hour live interactive training session for both pharmacists and technicians.

Methods: A one group pre-post intervention design study was conducted in 20 community-based pharmacies in a district of a large national community pharmacy chain. A one-hour live, interactive session was developed based on incident reports and medication error trends including medication error definition, ways in which medication errors occur, strategies for mitigating errors, information about human error, and methods and habits to improve patient safety. At least $50 \%$ of full-time staff for each of the 20 pharmacies were required to attend a training session between December 1, 2017-January 15, 2018. Participants completed a demographics survey documenting gender, age, credentials, number of years of experience, and years of service with the company. Pharmacies were categorized as low, medium or high volume based on prescription count. Medication errors were compared six months pre-and postlive education session. Data were analyzed using SAS version 9.4.

Results: One hundred and thirty-five errors and 111 errors were reported pre-and post-live training (mean 6.85 and 5.55, $p<0.301$ ), respectively. Nine pharmacies were low, eight medium, and three high volume with the mean number of medication errors reported pre-and post-live training; low 4.33 vs $4.11 p<0.478$, medium 9.37 vs $6.87 p<0.443$, and high 7.66 vs $6.33 p<0.593$, respectively. Sixty pharmacy staff (34 pharmacists, 26 technicians) attended one of the live training sessions; 73\% female; most frequently reported age range 35-50 years; 23 Doctor of Pharmacy, 11 Bachelor of Science in Pharmacy, and 26 Certified Technician; and average number of years' experience was 13.6 and average service with the company was 8.6 years.

Conclusions: A live training session for both pharmacists and technicians did not significantly decrease medication errors, but could be incorporated as an element of a medication safety program. It should be considered for implementation in other districts of this large national pharmacy chain as a part of an improved patient safety effort.

Key Words: medication errors, live session, patient safety, community pharmacy

\section{Background}

It is estimated that approximately 10 percent of all United States (U.S.) deaths are due to medical errors. This ranks as the $3^{\text {rd }}$ highest cause of death in the United States. ${ }^{1}$ Medication errors contribute significantly to medical error with approximately 50 million medication errors each year in the U.S. ${ }^{2}$ A medication error by definition is "Any preventable event that may cause or lead to inappropriate medication use or patient harm while the medication is in the control of the health care professional, patient, or consumer." ${ }^{3}$ In community pharmacies, a national observational study found a prevalence of prescription dispensing errors to be $1.7 \%$, equivalent to approximately 2 clinically significant errors per week. ${ }^{4}$ Common causes for medication errors in community pharmacies are medications that sound or look alike, lack of concentration due to interruptions, and lack of effective control of prescription, label, and medicine. ${ }^{5}$ The Institute of Safe

Corresponding author: Jean-Venable "Kelly" R. Goode, PharmD, BCPS, FAPhA, FCCP

Virginia Commonwealth University School of Pharmacy

PO Box 980533, Richmond, VA 23298-0533

Phone: (804) 828-3865; Fax: (804) 828-8359

E-mail: irgoode@vcu.edu
Medication Practices (ISMP) also provides insight and understanding how medication errors occur in the pharmacy setting and where errors occur including through order communication, product labeling, packaging, compounding, dispensing, distribution, administration, education, or monitoring malfunction or negligence. ${ }^{6}$ Furthermore, errors may be commission or omission in any stage of the medication use process. $^{7}$

Medication errors are multifactorial, largely involving more than just one breakdown in the process. Medication errors begin with faulty systems (latent conditions) that allow humans to fail (active failures) which occur through evolved mechanisms in the brain to handle the overload of information; during the day our brain creates adaptations to this overload as a means to keep up with the speed in which we are asking for information to be processed. ${ }^{8-10}$ Even with well-trained individuals, a lack of cognitive focus to keep up with the demand of daily work flow tasks is inevitable; this, as well as external factors play a role in medication errors.

ISMP identifies ten key elements in pharmacy work flow where there is an opportunity to prevent an error. ${ }^{7}$ The more defense/layers there are between the patient receiving the medication, the better. Also, fewer and smaller "holes" or lapses in work flow are more likely to allow errors to be caught. 
ISMP has identified the areas where defense may be prone to fail and contribute to medication errors and leaving out any one element or aspect can cause a medication error to happen. Errors can be minimized by following the standardized processes set by ISMP, engaging in continuing education and creating a fair and just culture in our pharmacies. Some strategies that must be implemented within pharmacy workflow include the following: active reduction of clutter and distractions (e.g., phones, televisions and open access to the internet), bar code scanning, drug utilization review for each prescription, a two-step verification process, prescription post fill auditing, and built in technologies that provide alerts when medication may be incorrect. ${ }^{6}$

Medication error management training is still evolving and may be in the form of live sessions, coaching or home study. Currently there are only a couple of states that require live medication error continuing education. ${ }^{11}$ For instance, as a biennial license renewal in Florida, The Florida State Board of Pharmacy requires pharmacists to complete a two hour course that contains specific components related to medication errors. ${ }^{12}$ Even with these requirements there is limited evidence that live continuing education can prevent medication errors. In a survey evaluating pharmacist's confidence on pediatric weight based dosing; pharmacists who received live training reported increased confidence with ensuring their pediatric patients received correct doses and thus reducing potential medication errors. ${ }^{13}$ Additionally, there is a gap in literature for assessment of pharmacists and technicians participating in a live training session together. This study will evaluate one strategy to prevent medication errors, the person approach, through developing a live training session for education of both pharmacists and technicians. Communitybased pharmacists and technicians learning together to understand factors that contribute medication errors and how to better work as a team should be well-positioned to prevent medication errors.

\section{Objective}

The objective is to compare community-based pharmacy medication errors before and after a one-hour live interactive training session for both pharmacists and technicians.

\section{Methods}

In this one group pre-post intervention study, six months of medication incident reports from 20 community pharmacies in a district of a large national community pharmacy chain spanning Virginia, as well as parts of Tennessee and West Virginia, were analyzed before and after a live one-hour interactive training session to determine the number of medication errors. The national large community pharmacy chain utilizes a standardized process with an internal electronic reporting system requiring error reporting, within 24 hours of discovery. Near misses are not required to but are encouraged to be reported as well.
A team of pharmacists who work for the large community pharmacy chain developed a live one-hour Accreditation Council for Pharmacy Education (ACPE) accredited education to address the three most common medication errors from the previous six months. The three most common medication errors reported occurred at reception, product dispensing and verification. The session included both team based interactive and lecture components. Topics addressed during the session included physical and mental fatigue, work place cohesiveness, ways in which medication errors occur, strategies for mitigating errors as well as pharmacy work flow best practices and current policies and procedures within the company. Each topic was taught in eight minute sections to compose the lecture portion. After completion of lecture, teams of four with both pharmacists and technicians applied the knowledge to discuss real world examples of how they could improve or change their current habits to reduce possible medication errors.

The same team of pharmacist(s) delivered this material between December 1, 2017 and January 15, 2018. A total of six live sessions were offered with a minimum of $50 \%$ of fulltime staff for each of the 20 pharmacies required to attend the session. Staffing levels at the time of the study were 80 pharmacists and 150 technicians, including certified and uncertified technicians, however only full time equivalent employees were required to attend. Additionally, demographics including gender, credentials and number of years of experience, years of service with Kroger and age were collected from a survey completed during the live session. Pharmacies were categorized as low, medium or high volume based on the average number of prescriptions filled per week and the average number of full time equivalents were documented for the pharmacies.

Medication errors are required to be recorded by the pharmacist who discovers the error via the online portal utilized by the national large pharmacy chain within 24 hours of discovering the incident. However, the patient must be contacted within the same business day. Additionally, near misses, defined as errors that occurred but never left the pharmacy are encouraged to be reported, but are not required. Any medication error incident reports submitted on a daily basis for six months before and after completion of the live session, were analyzed to determine if there was a significant reduction in the number of medication errors. Results were analyzed using a descriptive statistics platform, SAS version 9.4 and non-parametric statistical tests. A Wilcoxon signed rank test was used to compare if medication errors differed pre- and post-live training across all pharmacies and then if there was a difference if the pharmacy was low, medium and high volume. A Kruskal-Wallis test used to evaluate if the number of pharmacists and technicians differed across the low, medium and high volume pharmacies. The study was approved by the Virginia Commonwealth University Institutional Review Board. 


\section{Results}

Characteristics of the 20 community pharmacies included in the study are documented in Table 1 . The requirement of at least $50 \%$ of fulltime staff attendance was achieved with $53 \%$ of fulltime staff in attendance. Education level for attendees included 23 Doctors of Pharmacy, 11 Bachelors of Pharmacy and 26 certified technicians. Of those, $73 \%$ were female with the most frequent age range of 35 to 50 years of age. The pharmacy staff had worked in the pharmacy industry for an average of 13.6 years and had experience with this large community pharmacy chain for an average of 8.6 years. In the six months prior to the live training there were 137 medication errors reported and in the six months after 111 medication errors. While there was decrease in the number of errors (overall and within the three store type groups), the decrease was not statistically significant (overall $p<0.301$, low volume $p<0.478$, medium volume: $p<0.443$, high volume $p<0.593$, respectively). Comparisons were made between the number of pharmacists across pharmacies by the volume. The number of pharmacists significantly $(p<0.001)$ differed across the low, medium, and high volume pharmacies. Similarly, the number of technicians also significantly $(p<0.004)$ differed across the three pharmacy types.

\section{Discussion}

Patient safety is at the pinnacle of our profession. Pharmacists must have a continuous quality improvement process in place to provide the safest, most time efficient services. Additionally, pharmacists should engage in continuous professional development to improve the medication use process. There is limited research focused on medication errors in the community-based pharmacy setting. Furthermore, there is lack of published research within the past 5 years on medication errors occurring in community-based pharmacy with the advent of other patient care services. Reason states that human error can be viewed in two ways, 'the person approach and the system approach' understanding how each contribute to errors is an important aspect in improving and preventing medication errors. ${ }^{10}$

This study evaluated if the person approach, the live training session for both pharmacists and technicians reduced medication errors in a national large chain pharmacy setting. This study was also able show a decrease in medication errors during the study period post live training intervention. Additionally, errors found in this study were less than the two clinically significant errors per week per pharmacy found by Flynn and colleagues in a national observational study. ${ }^{4}$ Another study that conducted a live training session was able to document that pharmacist confidence improved and thus preventing potential medication errors in pediatric dosing. ${ }^{13}$ While this study did not measure confidence, it is inherent that more education should increase confidence.

Implementing a standard live training session for both pharmacists and technicians helped to streamline best practices within the pharmacy and reiterated the importance of patient safety through medication error reduction. The live training session played a role in re-training and establishing best practice protocols through an increased knowledge of policies and procedures. ${ }^{14} \mathrm{~A}$ similar study conducted in New Hampshire found that implementation of a formal certification program to ensure the consistency in training quality could assist in decreasing medication errors and improving patient safety. ${ }^{15}$ In another study conducted in the United Kingdom where work-related stress among community pharmacists was correlated with pharmacists' psychological and physical wellbeing, the occurrence of dispensing errors and detection of prescribing errors revealed that pharmacists reported significantly higher levels of workplace stressors than the general working population which highlights the need for ongoing education and continuous evaluation of the work environment and the systems used for dispensing as there is a correlation to dispensing errors, and detection of prescribing errors. ${ }^{16} \mathrm{~A}$ live training program that includes both pharmacists and technicians to discuss medication errors as team has the potential to reduce workplace stress with all team members understanding roles to reduce medication errors.

Additional studies will be needed to demonstrate if medication error rates are directly correlated to dispensing volume however, implementation of regular continuing education courses for license renewal may assist in knowledge of medication error definition, ways to mitigate medication errors as well as methods within workflow to catch errors before they reach the patient. The large community pharmacy chain was able to see increased awareness however, future studies should evaluate the correlation between faulty systems (latent conditions) that allow humans to fail (active failures) focusing on the systems to help reduce the failing of humans.

\section{Limitations}

The generalizability of the study to all pharmacists in community-based practice is limited due to convenience sampling and the focus on a district of pharmacies within a large community pharmacy chain. Additionally, there is potential for low retention of the information presented at the live training session and not all pharmacy staff attended the live training session. It is not known which pharmacists were involved in the medication errors post-live training. Medication error reduction may be hard to attribute solely to the intervention as maturation of pharmacists and technicians may have also played a role in the reduction of errors outside of the live training session. Additionally, using event reporting data is generally accepted but flawed as it relies on someone to fill out a report.

This district of the large community pharmacy chain holds medication safety meetings each quarter to address any outstanding or egregious errors. During these meetings, changes in policies and procedures and advances in best practices related to medication errors may be implemented in order to keep medication error rates as low as possible. At the 
same time this project was started, these meetings were restructured to create a more action driven atmosphere in the pharmacy and may be a limitation regarding the true outcomes of the study. Additionally, proprietary limitations influenced the amount and type of data that could be shared, some of which may have provided better insight.

\section{Conclusions}

A live training session for both pharmacists and technicians did not significantly decrease medication errors, but could be incorporated as an element of a comprehensive medication safety program. It should be considered for implementation in other districts of this large national pharmacy chain as a part of an improved patient safety effort. Continuing education and knowledge about medication errors plays an important role in accuracy and patient safety and educating staff about medication safety is foundational.

Acknowledgements: Michele Fountain, PharmD, Mid-Atlantic Regional Clinical Manager of Kroger Pharmacy

Funding: This research did not receive any specific grant from funding agencies in the public, commercial, or not-for-profit sectors.

Conflicts of Interest: We declare no conflicts of interest or financial interests that the authors or members of their immediate families have in any product or service discussed in the manuscript, including grants (pending or received), employment, gifts, stock holdings or options, honoraria, consultancies, expert testimony, patents and royalties.

Treatment of Human Subjects: Virginia Commonwealth University IRB Exemption granted.

\section{References}

1. Makary Ma, Daniel M.

BMJ 2016; 353;i2139. https://doi.org/10.1136/bmj.i213 9 Accessed July 10, 2020.

2. Aspden $\mathrm{P}$, Wolcott JA, Bootman JL, and Cronenwett, LR, eds. (2007). Preventing Medication Errors. Washington, DC: The National Academies Press.

http://nap.edu/11623 Accessed July 10, 2020.

3. National Coordinating Council for Medication Error Reporting and Preventing. About Medication Errors. Accessed http://www.nccmerp.org/about-medicationerrors. Accessed July 10, 2020.

4. Flynn EA, Barker KN, Carnahan BJ. National observational study of prescription dispensing accuracy and safety in 50 pharmacies. J Am Pharm Assoc 2003;43(2):191-200

5. Knudsen $P$, Herborg $H$, Mortensen AR, Knudsen $M$, and Hellebek A. Preventing medication errors in community pharmacy: root-cause analysis of transcription errors. Qual Safe Health Care 2007;16(4), 285-290.

6. Institute for Safe Medication Practices. Key Elements of Medication Errors https://www.ismp.org/ten-keyelements. Accessed July 10, 2020.

7. Discern Health. Addressing Medication Safety in Community Pharmacy. http://discernhealth.com/wpcontent/uploads/2018/07/Medication-SafetyCommunity-Pharmacy-White-Paper-2018-02-27.pdf Accessed July 10, 2020.

8. Grissinger M. Inattentional Blindness: What Captures Your Attention? Pharmacy and Therapeutics. 2012;37(10):542-555

9. Medication Errors. PSNet: Patient Safety Network https://psnet.ahrq.gov/primers/primer/23/medicationerrors. Accessed September 15, 2018.

10. Reason J. Human error: models and management. BMJ. 2000;320(7237):768-70.

11. CE For pharmacists: Find your state's requirements. https://universitylearning.com/blog/medicalnews/2016/12/18/ce-pharmacists-find-statesrequirements/ Accessed July 10, 2020

12. Pharmacists, F. (n.d.). Continuing Education Resources Florida Society of Health-System Pharmacists. https://www.fshp.org/page/CEresources Accessed July 10, 2020.

13. Condren M E,Desselle,S P. The Fate of Pediatric Prescriptions in Community Pharmacies. J Patient Saf 2015;11(2), 79-88. doi:10.1097/pts.0b013e3182948a7d

14. Kroger Health Best Practices. Internal Documents. 2018.

15. Pervanas HC, Revell N, Alotaibi AF. Evaluation of Medication Errors in Community Pharmacy Settings. J Pharm Technol 2015;32(2): 71-74

16. Johnson SD, O'Connor EM, Jacobs S, Hassell K, Ashcroft $D M$. The relationships among work stress, strain and reported errors in UK community pharmacy. Res Soc Adm Pharm Pharmacy 2014;10:885-895 
Table 1. Pharmacy Characteristics

\begin{tabular}{|c|c|c|}
\hline Pharmacy & Script count & $\begin{array}{c}\text { Mean Number of full time Pharmacists and } \\
\text { Technicians (Standard Deviation) }\end{array}$ \\
\hline Low volume- 9 & $<1500$ per week & $\begin{array}{c}\text { Pharmacists:2 (0) } \\
\text { Certified Technicians: } 1 \text { (0.52) }\end{array}$ \\
\hline Medium volume- 8 & $1500-3000$ per week & $\begin{array}{l}\text { Pharmacists: } 2 \text { (0.35) } \\
\text { Certified Technicians: } 2 \text { (0.35) }\end{array}$ \\
\hline High volume- 3 & >3000 per week & $\begin{array}{l}\text { Pharmacists: } 3(0) \\
\text { Certified Technicians: } 4 \text { (0) }\end{array}$ \\
\hline
\end{tabular}

Table 2. Medication Errors

\begin{tabular}{|c|c|c|}
\hline Weekly Script Count & $\begin{array}{c}\text { Mean medication errors pre-live training } \\
\text { session (standard deviation) }\end{array}$ & $\begin{array}{c}\text { Mean medication errors post-live } \\
\text { training session (standard deviation) }\end{array}$ \\
\hline Overall & $6.85(6.50)$ & $5.55(4.97)$ \\
\hline Low Volume & $4.33(6.50)$ & $4.11(4.97)$ \\
\hline Medium Volume & $9.37(8.53)$ & $6.87(6.49)$ \\
\hline High Volume & $7.66(7.02)$ & $6.33(0.57)$ \\
\hline
\end{tabular}

